\title{
A DETECTION OF THE INTEGRATED SACHS-WOLFE IMPRINT OF COSMIC SUPERSTRUCTURES USING A MATCHED-FILTER APPROACH
}

\author{
SEShadri NADATHur AND Robert CRitTEnden \\ Institute of Cosmology and Gravitation, University of Portsmouth, Burnaby Road, Portsmouth PO1 3FX, UK; seshadri.nadathur@port.ac.uk \\ Received 2016 August 24; revised 2016 September 21; accepted 2016 September 23; published 2016 October 11
}

\begin{abstract}
We present a new method for detection of the integrated Sachs-Wolfe (ISW) imprints of cosmic superstructures on the cosmic microwave background (CMB), based on a matched-filtering approach. The expected signal-to-noise ratio for this method is comparable to that obtained from the full cross-correlation, and unlike other stacked filtering techniques it is not subject to an a posteriori bias. We apply this method to Planck CMB data using voids and superclusters identified in the CMASS galaxy data from the Sloan Digital Sky Survey Data Release 12, and measure the ISW amplitude to be $A_{\mathrm{ISW}}=1.64 \pm 0.53$ relative to the $\Lambda \mathrm{CDM}$ expectation, corresponding to a $3.1 \sigma$ detection. In contrast to some previous measurements of the ISW effect of superstructures, our result is in agreement with the $\Lambda$ CDM model.
\end{abstract}

Key words: cosmic background radiation - cosmology: observations - cosmology: theory - dark energy - largescale structure of universe

\section{INTRODUCTION}

Since the discovery of the accelerated expansion of the universe (Riess et al. 1998; Perlmutter et al. 1999), the nature of dark energy has become one of the central puzzles in cosmology. The time evolution of gravitational potentials produces secondary temperature anisotropies in the cosmic microwave background $(\mathrm{CMB})$ via the late-time integrated Sachs-Wolfe (ISW) effect (Sachs \& Wolfe 1967). Because this time evolution can be caused by a cosmological constant $\Lambda$, measurement of the ISW signal is a direct probe of the dynamical effects of dark energy (Crittenden \& Turok 1996).

The ISW signal is conventionally measured through a crosscorrelation between the CMB and large-scale structure (LSS) tracers (e.g., Fosalba et al. 2003; Afshordi 2004; Boughn \& Crittenden 2004; Nolta et al. 2004; Giannantonio et al. 2006, 2008; Ho et al. 2008). This method yields typical detection significances of $2 \sigma-3 \sigma$ for individual LSS tracers, rising to $\sim 4 \sigma$ through a combination of multiple tracers (Giannantonio et al. 2012; Planck Collaboration et al. 2016b).

An alternative method for detection is to stack filtered CMB patches around the locations of localized "superstructures"large empty cosmic voids and overdensities known as superclusters. Granett et al. (2008) used this method with WMAP CMB data and 100 superstructures from SDSS to report a $\sim 4.5 \sigma$ detection, subsequently confirmed with Planck data (Planck Collaboration et al. 2014, 2016b).

However, this detection has proven difficult to interpret, due to the large amplitude of the observed effect. Theoretical estimates with optimistic assumptions (Nadathur et al. 2012; Flender et al. 2013; Aiola et al. 2015) showed that it exceeds the maximum expectation in a $\Lambda \mathrm{CDM}$ cosmology by a factor of $\sim 5$, a $\gtrsim 3 \sigma$ discrepancy with theory. Simulation results give an even smaller expected signal (Cai et al. 2014; Hotchkiss et al. 2015), exacerbating the problem.

Efforts to replicate the measurement with independent superstructure catalogs have either given null results (Ilić et al. 2013; Hotchkiss et al. 2015) or marginally significant detections with amplitude still in excess of expectation (Cai et al. 2014; Granett et al. 2015; Kovács \& Granett 2015). Such studies often rely on arbitrary choices for the number of superstructures included in the stacks and the width of the compensated top-hat (CTH) filter used in the analysis, potentially introducing important a posteriori biases in the analysis (Hernández-Monteagudo \& Smith 2013).

In this study, we describe a new method for the detection of the stacked ISW signal using matched filters constructed after calibration on simulations. Our method has a far higher expected sensitivity, even comparable with that expected from the full cross-correlation technique, and is free of possible a posteriori bias. We applied this method to data from the CMASS galaxy sample from SDSS Data Release 12 (DR12) and Planck, and report a detection of the ISW effect of superstructures at $3.1 \sigma$ significance. The amplitude of the ISW effect is consistent with $\Lambda \mathrm{CDM}$ expectations, thus potentially resolving this long-standing anomaly.

\section{DATA SETS}

\subsection{LSS Data and Simulations}

We identified cosmic voids and superclusters in the CMASS galaxy sample of the SDSS-III BOSS DR12 (Alam et al. 2015). ${ }^{1}$ This is the final data release of SDSS-III. The BOSS LSS galaxy catalogs provide spectra and redshifts for 1.3 million galaxies over $9,376 \mathrm{deg}^{2}$ in two contiguous sky regions in the Northern and Southern Galactic Caps. The CMASS sample includes 777202 luminous galaxies in the redshift range $0.43 \leqslant z \leqslant 0.7$ and is selected to be approximately volume-limited in stellar mass. Details on the target selection, data reduction algorithms, and catalog creation are given in Reid et al. (2016).

To find voids and superclusters we used a modified version of the ZOBOV algorithm (Neyrinck 2008), following Nadathur (2016). ZOBOV reconstructs the local galaxy density field from the discrete galaxy distribution using a Voronoi tessellation, identifies local extrema of the density field, and then uses a watershed algorithm to demarcate individual structures. To prevent the tessellation from leaking beyond the

\footnotetext{
http://data.sdss3.org/sas/dr12/boss/lss/
} 
observed survey volume we add a thin layer of buffer particles around the boundary of the survey footprint, within holes in the survey mask, and along both the high- and low-redshift caps (see Nadathur 2016). The volumes of the Voronoi cells associated with each galaxy are inverted to estimate local tracer densities, after applying a redshift- and position-dependent weighting to account for variations in the survey mean density $n(z)$ and the survey sky completeness.

Our implementation of the watershed algorithm for voidfinding followed that of Nadathur (2016). In particular, we did not merge neighboring voids together, separating individual structures purely on the basis of the underlying topology of the density field. For superclusters, we applied the same voidfinding algorithm to the inverse of the density field, thus identifying density maxima instead of minima. For each superstructure, we determined the average galaxy density contrast, $\bar{\delta}_{g}=\frac{1}{V} \int_{V} \delta_{g} d^{3} \boldsymbol{x}$, and effective spherical radius, $R_{\text {eff }}=\left(\frac{3}{4 \pi} V\right)^{1 / 3}$, where the superstructure volume $V$ is determined from the sum of the volumes of Voronoi cells making up the structure. The center of each void is defined as the center of the largest completely empty sphere that can be inscribed within it (Nadathur \& Hotchkiss 2015a; Nadathur 2016). For superclusters, we took the location of the galaxy with the smallest Voronoi cell within the supercluster as its center.

For calibration of the expected ISW signal from voids and superclusters, we compared the gravitational potential information in the Big MultiDark (BigMD) $N$-body simulation (Klypin et al. 2016) with superstructures found in a mock CMASS galaxy catalog created in that simulation (for details see $\mathrm{S}$. Nadathur et al. 2016, in preparation). Previous work using this simulation has shown that such voids and superclusters correspond to large but relatively shallow matter density perturbations within the linear or quasi-linear regime, extending over scales of up to $\mathcal{O}\left(100 h^{-1} \mathrm{Mpc}\right)$ (Nadathur \& Hotchkiss 2015a, 2015b; S. Nadathur et al. 2016, in preparation).

To test the operation of our algorithm on CMASS data and to estimate error covariances, we applied the same structurefinding procedure to 1000 sets of mock galaxy catalogs created using the "quick particle mesh" (QPM) technique (White et al. 2014). These mocks are based on a set of low-resolution particle mesh simulations consisting of $1280^{3}$ particles in a box of side $2.56 \mathrm{~h}^{-1} \mathrm{Gpc}$, with cosmological parameters $\Omega_{\mathrm{m}}=0.29, h=0.7, n_{s}=0.97$, and $\sigma_{8}=0.8$. Halos in the simulations are populated with galaxies using a halo occupation distribution method to reproduce the observed galaxy clustering amplitude. These mocks also incorporate observational effects of the survey selection, veto mask, and fiber collisions. The distribution of superstructures in the QPM mocks revealed some localized residual effects of the survey boundary on the tessellation near some of the holes in the survey mask. All superstructures in these regions were treated as contaminated and removed from both the CMASS catalogs and the mocks.

The final catalog of structures used in the analysis has been made available for download. ${ }^{2}$

\footnotetext{
http://www.icg.port.ac.uk/stable/nadathur/voids/
}

\section{2. $C M B$ Data}

We used the four foreground-cleaned CMB temperature maps from the Planck 2015 data release. These are the COMMANDER, NILC, SEVEM, and SMICA maps, named after the component separation methods used to generate them (Planck Collaboration et al. 2016a). The maps are pixelized in HEALPix format (Gorski et al. 2005), at resolution $N_{\text {side }}=1024$, corresponding to a mean pixel spacing of 3.4 arcminutes. To these maps we applied the common Planck UT78 temperature mask, downgraded to the same $N_{\text {side }}$ resolution using a binary threshold cut of 0.9 , to eliminate contamination from the Galactic plane and known point sources.

\section{METHOD}

\subsection{Constructing Template Profiles}

The ISW temperature shift along direction $\boldsymbol{n}$ is given by the line-of-sight integral

$$
\frac{\Delta T_{\mathrm{ISW}}}{\bar{T}}(\hat{\boldsymbol{n}})=2 \int_{0}^{z_{\mathrm{LS}}} \frac{a}{H(z)} \dot{\Phi}(\hat{\boldsymbol{n}}, \chi(z)) d z,
$$

where the integral extends to the redshift of last scattering, $z_{\mathrm{LS}}$. In the linear approximation, density perturbations grow as $\dot{\delta}=\dot{D} \delta$, where $D(z)$ is the linear growth function. This can be combined with the Poisson equation for $\Phi$ to obtain

$$
\frac{\Delta T_{\mathrm{ISW}}}{\bar{T}}(\hat{\boldsymbol{n}})=-2 \int_{0}^{z_{\mathrm{LS}}} a(z)(1-f(z)) \Phi(\hat{\boldsymbol{n}}, z) d z,
$$

where $f=\frac{d \ln D}{d \ln a}$ is the growth rate. This linear approach is an extremely good approximation on the scales of interest (e.g., Cai et al. 2010; Nadathur et al. 2014).

The ISW temperature profile produced by a given structure can be calculated using knowledge of the gravitational potential $\Phi(r)$ about its location, which must be determined from calibration with the simulation. We follow the results of S. Nadathur et al. (2016, in preparation), who studied structures identified using a mock galaxy catalog in the BigMD simulation, finding that the value of $\Phi$ at void locations is tightly correlated with the observable quantity

$$
\lambda_{v} \equiv \bar{\delta}_{g}\left(\frac{R_{\mathrm{eff}, v}}{1 h^{-1} \mathrm{Mpc}}\right)^{1.2} \text {. }
$$

The majority of voids identified by ZOBOV correspond to local underdensities within globally overdense regions and thus do not give $\Delta T_{\text {ISW }}<0$. However, those voids with $\lambda_{v}<0$ are on average undercompensated, corresponding to regions with $\Phi>0$ and thus a negative ISW shift. For such voids, S. Nadathur et al. (2016, in preparation) find that the spherically averaged potential profile at distance $r$ from the void center follows the two-parameter form

$$
\bar{\Phi}\left(r, \lambda_{v}\right)=\frac{\Phi_{0 v}\left(\lambda_{v}\right)}{1+\left(r / r_{0 v}\left(\lambda_{v}\right)\right)^{2}},
$$

with $\Phi_{0 v}\left(\lambda_{v}\right)$ and $r_{0 v}\left(\lambda_{v}\right)$ calibrated from fits to simulation.

We examined the properties of superclusters in the same BigMD simulation and found an analogous result. For 

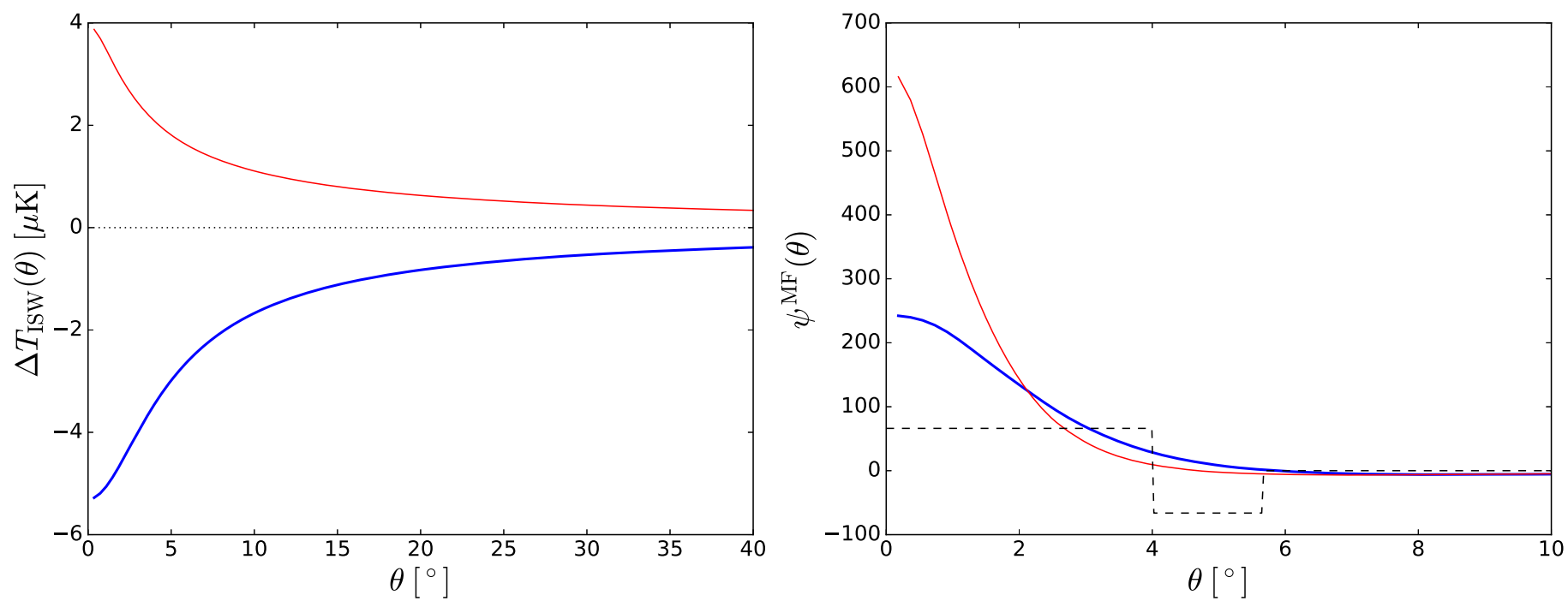

Figure 1. Left: template ISW temperature profiles calculated for a void with $\lambda_{v}=-36.9$ (thick blue line) and a supercluster with $\lambda_{c}=272.4$ (thin red), both assumed to be centered at redshift $z=0.55$. Right: optimal matched filters constructed for these two templates using Equation (11). For comparison the dashed black line shows a CTH filter of width $4^{\circ}$, as used by Granett et al. (2008).

superclusters, the observable

$$
\lambda_{c} \equiv \bar{\delta}_{g}\left(\frac{R_{\mathrm{eff}, c}}{1 h^{-1} \mathrm{Mpc}}\right)^{1.6}
$$

is an excellent empirical predictor for the value of $\Phi$. Superclusters with $\lambda_{c}>0$ on average correspond to $\Phi<0$ and thus $\Delta T_{\text {ISW }}>0$. We found the average potential profile for these structures followed

$$
\bar{\Phi}\left(r, \lambda_{c}\right)=\frac{\Phi_{0 c}\left(\lambda_{c}\right)}{1+\left(r / r_{0 c}\left(\lambda_{c}\right)\right)^{\alpha\left(\lambda_{c}\right)}},
$$

with $\Phi_{0 c}\left(\lambda_{c}\right), r_{0 c}\left(\lambda_{c}\right)$, and $\alpha\left(\lambda_{c}\right)$ again determined from a simulation. Importantly, the amplitude of the potential fluctuation scales linearly with both observables, $\Phi_{0 v, c} \propto-\lambda_{v, c}$ (see also S. Nadathur et al. 2016, in preparation). The length scales of the potential perturbations, $r_{0 v}$ and $r_{0 c}$, typically far exceed the physical extents of the voids and superclusters, as expected from the Poisson equation.

We used the fitted profile forms from Equations (4) and (6), with Equation (2), to calculate the expected ISW temperature shift $\Delta T_{\text {ISW }}(\theta)$ at angle $\theta$ from the line of sight to a given void or supercluster, located at a given redshift. This can be split into an amplitude and a spatial profile normalized to unity, as $\Delta T_{\text {ISW }}(\theta)=T_{0} y(\theta)$. The axisymmetric template profile can be expanded into spherical harmonics, as

$$
y(\theta)=\sum_{\ell=0}^{\infty} y_{\ell 0} Y_{\ell}^{0}(\cos \theta)
$$

\subsection{Matched-filter Construction}

The total temperature signal at sky location $\boldsymbol{\theta}=(\vartheta, \varphi)$ can be written as

$$
s(\boldsymbol{\theta})=\Delta T_{\mathrm{ISW}}\left(\left|\boldsymbol{\theta}-\boldsymbol{\theta}_{0}\right|\right)+n(\boldsymbol{\theta}),
$$

where $\Delta T_{\text {ISW }}$ is the template ISW contribution calculated above, $\boldsymbol{\theta}_{0}$ is the location of the structure center, and $n(\boldsymbol{\theta})$ includes all other sources of noise in the foreground-cleaned maps, to which the dominant contribution comes from primordial CMB fluctuations at the last scattering surface. To isolate the ISW signal from the noise, we apply an axisymmetric matched-filter $\psi^{\mathrm{MF}}(\theta)$ to the observed map. The resulting filtered map $u(\boldsymbol{\beta})$ is a convolution of the filter function and the observed map,

$$
u(\boldsymbol{\beta})=\int d \Omega s(\boldsymbol{\theta}) \psi^{\mathrm{MF}}(|\boldsymbol{\theta}-\boldsymbol{\beta}|) .
$$

The coefficients of the spherical harmonic expansion of the filtered map can be written as (Schäfer et al. 2006)

$$
u_{\ell m}=\sqrt{\frac{4 \pi}{2 \ell+1}} s_{\ell m} \psi_{\ell 0}^{\mathrm{MF}}
$$

Given a template profile from Equation (7), the optimal matched-filter $\psi^{\mathrm{MF}}$ satisfies two constraints:

1. the expectation value of the filtered field at the structure location is an unbiased estimator of the ISW amplitude, $\left\langle u\left(\boldsymbol{\theta}_{0}\right)\right\rangle=T_{0}$, and

2. the variance of the filtered field is minimized.

These constraints are satisfied by choosing (Schäfer et al. 2006; McEwen et al. 2008)

$$
\psi_{\ell 0}^{\mathrm{MF}}=\kappa \frac{y_{\ell 0}}{C_{\ell}},
$$

where $\kappa^{-1}=\sum_{\ell=0}^{\infty} y_{\ell 0}^{2} / C_{\ell}$, and $C_{\ell}$ denotes the power spectrum multipoles of the noise field-in this case, the power spectrum of the Planck CMB maps.

We determined the appropriate choice of filter coefficients $\psi_{\ell 0}^{\mathrm{MF}}$ from the template $y_{\ell 0}$ and Equation (11). The filter depends on the fit parameters $r_{0 v}\left(\lambda_{v}\right)$ (for voids) or $r_{0 c}\left(\lambda_{c}\right)$ and $\alpha\left(\lambda_{c}\right)$ (for superclusters), and on the comoving distance to the structure center. The signal amplitude $T_{0}$ depends on all of these as well as on the amplitude of the potential fluctuation $\Phi_{0 v, c}$.

Figure 1 shows example templates $\Delta T_{\mathrm{ISW}}(\theta)$ and matchedfilter profiles $\psi^{\mathrm{MF}}(\theta)$ for a void with $\lambda_{v}=-36.9$ and a supercluster with $\lambda_{c}=272.4$. Both are assumed to be centered 

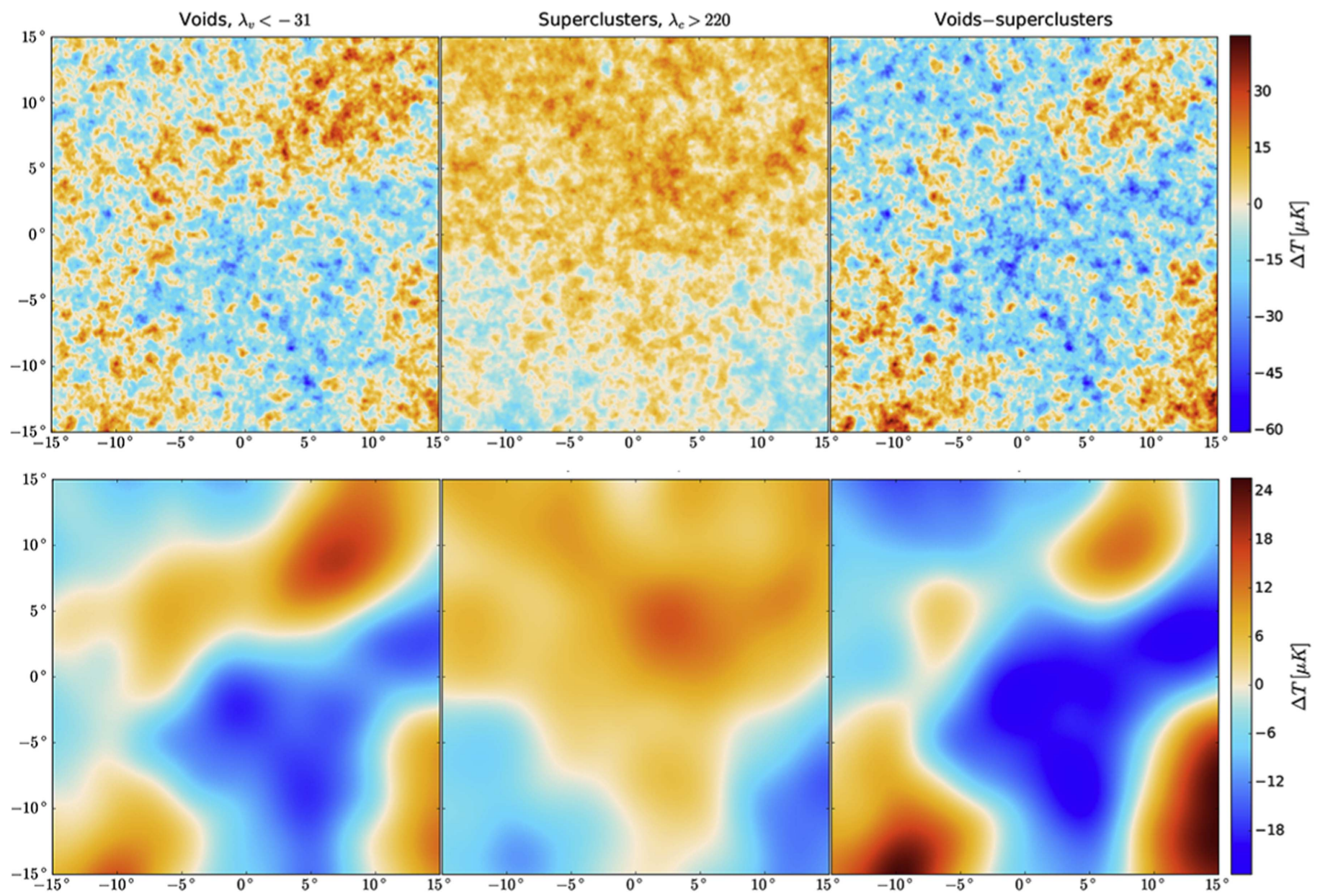

Figure 2. Top row: stacked regions of the unfiltered Planck SEVEM map centered on the positions of 96 voids with $\lambda_{v}<-31$ (left), 353 superclusters with $\lambda_{c}>220$ (center), and the difference between them (right). These correspond to the two extreme bins in Figure 3, and to the template profiles in Figure 1. Bottom row: same as above, but after filtering with the appropriate matched filters $\psi^{\mathrm{MF}}(\theta)$.

at redshift $z=0.55$. Also shown for comparison is a CTH filter, used by Granett et al. (2008) and other previous ISW stacking analyses.

All of our superstructure templates have expected $\mathrm{S} / \mathrm{N} \lesssim 0.1$. Therefore a detection is only possible by stacking large numbers of structures.

\subsection{Detection Strategy}

To measure the ISW effect of superstructures we first pruned the structure catalogs to remove all voids with $\lambda_{v}>0$ and superclusters with $\lambda_{c}<0$, as they are not expected to contribute an ISW shift with the right sign. This left a total of 2445 voids and 29,866 superclusters. We then binned the remaining structures according to their values of $\lambda_{v}$ and $\lambda_{c}$, respectively.

For each bin, we determined the average $\bar{\lambda}_{v}$ (or $\bar{\lambda}_{c}$ ) and the mean redshift of structures in the bin. From these and the fits to Equations (4) and (6) from simulations, we obtained the expected ISW amplitude $T_{0}$, the profile $y(\theta)$, and the appropriate matched-filter $\psi^{\mathrm{MF}}(\theta)$ for the representative template in each bin. Details of the fits to $\Phi(r)$ used are provided for download, together with the superstructure catalogs.

Note that we constructed the matched filters entirely based on calibration with simulations and before any reference to the
CMB data. No free parameters remain in our analysis, so it is free of any a posteriori bias.

We filtered the appropriately masked CMB maps with the matched filters and stacked the results at the locations of superstructures in each bin to obtain the average values $T_{0}^{\text {measured }}=\overline{u\left(\boldsymbol{\theta}_{0}\right)}$ in each case. To estimate errors we generated 2000 mock measurements by applying the same filters to random realizations of the CMB sky, at the locations of superstructures in randomly selected catalogs from the set of QPM mocks. We also tested generating mocks by keeping the superstructure catalog fixed but still varying the CMB maps, or by varying the catalog but fixing the Planck CMB map. These gave comparable results, but do not simultaneously capture both sources of variability.

\section{RESULTS}

Figure 2 shows stacked CMB map patches centered on voids and superclusters in the two extreme bins, containing structures with $\lambda_{v}<-31$ and $\lambda_{c}>220$, respectively. A visually compelling difference can be seen between the two stacks, although comparison with the templates in Figure 1 shows the difference in magnitude between the ISW signal and the primordial CMB noise, highlighting the need for a statistical approach. 

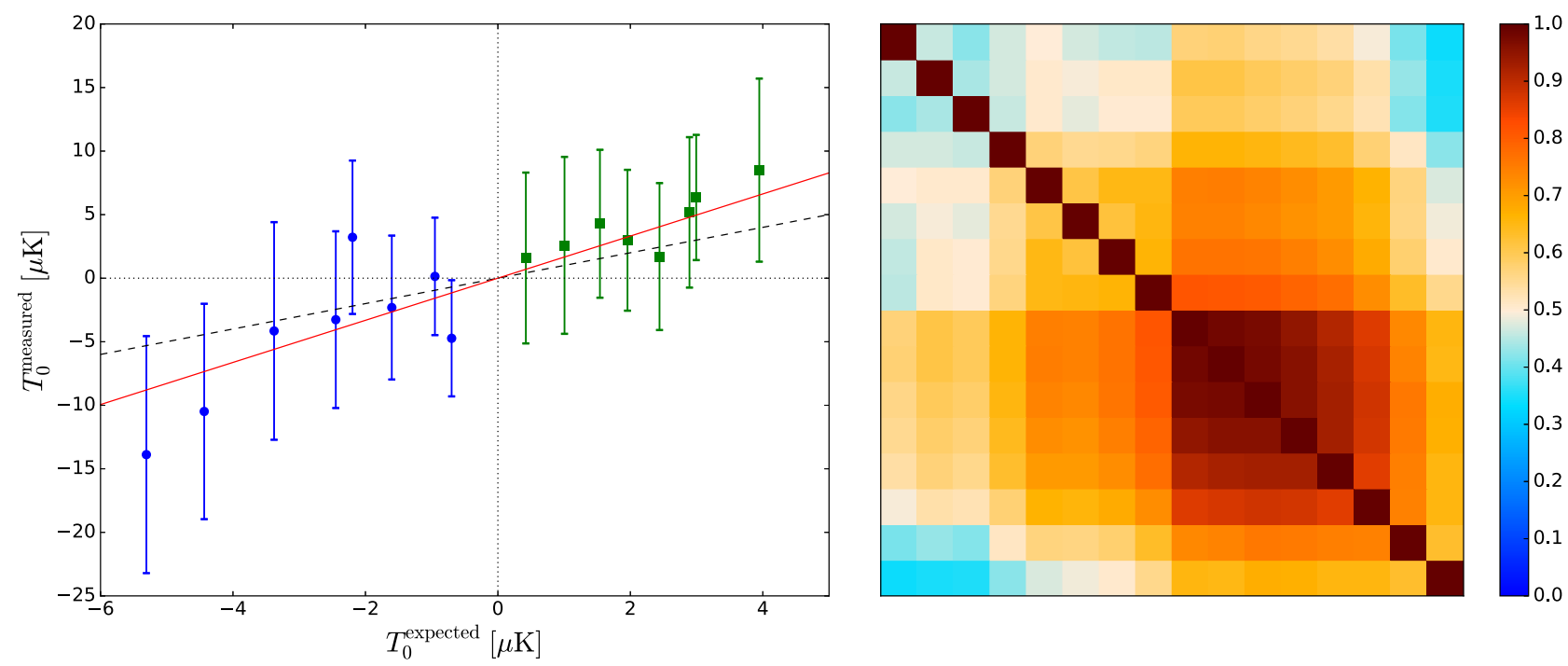

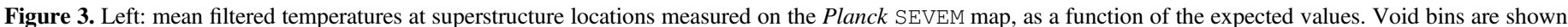

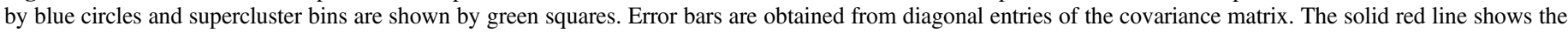

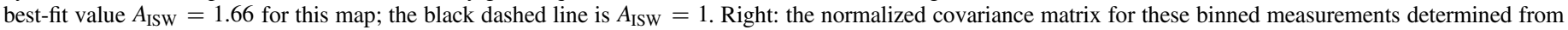
mock realizations.

In the left panel of Figure 3 we show the results obtained for $T_{0}^{\text {measured }}$ in each of the 16 bins for the SEVEM map, as a function of the expected ISW amplitude in the bin. Measurements in different bins are highly correlated with each other, as can be seen from the normalized covariance matrix shown in the right panel. We fitted a straight line of the form

$$
T_{0}^{\text {measured }}=A_{\mathrm{ISW}} T_{0}^{\text {expected }}
$$

to this data, where the free parameter $A_{\mathrm{ISw}}=1$ for a $\Lambda \mathrm{CDM}$ cosmology. The results obtained for each of the four $\mathrm{CMB}$ maps are summarized in Table 1. The likelihood function for $A_{\text {ISW }}$ is Gaussian in all cases. All four maps give very similar results, indicating a cosmological origin for the signal. A simple average of the results gives $A_{\mathrm{ISW}}=1.64 \pm 0.53$, with a signal-to-noise ratio of 3.1 .

For the same structures, using CTH filters with widths in each bin chosen to maximize the available signal from the template profiles gave $A_{\mathrm{ISW}}=1.54 \pm 0.73$, consistent with our headline result but with $38 \%$ larger uncertainty, due to the suboptimal filter choice.

\section{DISCUSSION}

We have presented a new method for detecting the ISW temperature shift in the CMB due to cosmic superstructures, using a combination of stacking and a matched-filter analysis calibrated on simulations. Applying this method to superstructures in the CMASS galaxy data, we obtain a measurement of the ISW amplitude, $A_{\mathrm{ISW}}=1.64 \pm 0.53$, significant at the $3.1 \sigma$ equivalent level. This value is insensitive to the method of foreground removal in the CMB maps, pointing to its cosmological origin. This detection significance is among the highest obtained for the ISW using any single LSS tracer (Giannantonio et al. 2012; Planck Collaboration et al. 2016b).

An important advantage of our new method is that all analysis choices and parameters have been fixed purely based on calibration with the simulation, before looking at the CMB data. This means that our measurement is not subject to any
Table 1

Measurements of the ISW Amplitude from Matched-filter Stacking Analysis with Planck and CMASS Superstructures

\begin{tabular}{lcc}
\hline \hline CMB map & $A_{\text {ISW }} \pm \sigma_{A}$ & $\mathrm{~S} / \mathrm{N}$ \\
\hline COMMANDER & $1.65 \pm 0.53$ & 3.13 \\
NILC & $1.62 \pm 0.53$ & 3.07 \\
SEVEM & $1.66 \pm 0.53$ & 3.15 \\
SMICA & $1.62 \pm 0.53$ & 3.08 \\
\hline
\end{tabular}

a posteriori bias. In contrast, several previous ISW stacking measurements on superstructures have included arbitrary choices of the number of superstructures and width of the CTH filter used, potentially affecting the claimed detection significances (Hernández-Monteagudo \& Smith 2013).

Our method also provides a greatly increased sensitivity over previous stacking analyses, such that we find an expected $\mathrm{S} / \mathrm{N}$ of 1.9 even for a standard $\Lambda \mathrm{CDM}$ cosmology. This is due to a combination of factors: better statistics due to the large size of our new catalog of superstructures; an improved calibration with the simulation allowing for a more optimal binning in $\lambda_{v}$ and $\lambda_{c}$; and the use of optimal matched filters in place of the $\mathrm{CTH}$ filters used in previous studies.

The value of $A_{\text {ISW }}$ that we obtain is larger than the $\Lambda \mathrm{CDM}$ expectation but is consistent with it at $1.2 \sigma$, similar to other results using luminous red galaxies in cross-correlation (e.g., Giannantonio et al. 2012). This is in contrast to the highsignificance detections of the stacked ISW signal reported by Granett et al. (2008) and Planck Collaboration et al. (2016b), which exceed the $\Lambda \mathrm{CDM}$ expectation by a factor of $\sim 5$ or more, corresponding to a $\gtrsim 3 \sigma$ discrepancy (see Nadathur et al. 2012; Flender et al. 2013; Cai et al. 2014; Aiola et al. 2015; Hotchkiss et al. 2015). Such a large discrepancy has been hard to explain in any alternative theoretical models. Our result is therefore an important step toward the resolution of this apparent anomaly.

Our result is also relevant to the proposed explanation of the CMB "Cold Spot" as being due to the ISW effect of a giant 
void (Szapudi et al. 2015). This would require a very large enhancement of $A_{\mathrm{ISW}}$ (Nadathur et al. 2014; Marcos-Caballero et al. 2016), which is not supported by our data.

Finally, it is also noteworthy that the ISW detection method presented here has a sensitivity similar to that of the traditional cross-correlation of projected galaxy density maps with the CMB. For comparison, Planck Collaboration et al. (2016b) reported an expected $\mathrm{S} / \mathrm{N}$ of 1.79 for the cross-correlation of the combined SDSS CMASS and LOWZ surveys with Planck, albeit based on a data release with smaller sky coverage and photometric redshifts. Our method allows precise measurements of the ISW effect and thus the dynamic effects of dark energy specifically in the extreme density environments of voids and superclusters. It will therefore be useful in further tests of $\Lambda$ CDM with future LSS data.

We thank Shaun Hotchkiss for useful discussions. S.N. acknowledges an Individual Fellowship of the Marie Skłodowska-Curie Actions under the H2020 Framework of the European Commission, project COSMOVOID.

This work has made use of public data from the SDSS-III collaboration. Funding for SDSS-III has been provided by the Alfred P. Sloan Foundation, the Participating Institutions, the National Science Foundation, and the U.S. Department of Energy Office of Science. The SDSS-III website is http:// www.sdss3.org/. The Big MultiDark simulations were performed on the SuperMUC supercomputer at the LeibnizRechenzentrum in Munich, using resources awarded to PRACE project number 2012060963. We acknowledge use of the EREBOS, THEIA, and GERAS clusters at the Leibniz Institut für Astrophysik (AIP).

\section{REFERENCES}

Afshordi, N. 2004, PhRvD, 70, 083536

Aiola, S., Kosowsky, A., \& Wang, B. 2015, PhRvD, 91, 043510

Alam, S., Albareti, F. D., Allende Prieto, C., et al. 2015, ApJS, 219, 12

Boughn, S., \& Crittenden, R. 2004, Natur, 427, 45

Cai, Y.-C., Cole, S., Jenkins, A., \& Frenk, C. S. 2010, MNRAS, 407, 201
Cai, Y.-C., Neyrinck, M. C., Szapudi, I., Cole, S., \& Frenk, C. S. 2014, ApJ, 786, 110

Crittenden, R. G., \& Turok, N. 1996, PhRvL, 76, 575

Flender, S., Hotchkiss, S., \& Nadathur, S. 2013, JCAP, 1302, 013

Fosalba, P., Gaztanaga, E., \& Castander, F. 2003, ApJL, 597, L89

Giannantonio, T., Crittenden, R., Nichol, R., \& Ross, A. J. 2012, MNRAS, 426, 2581

Giannantonio, T., Crittenden, R. G., Nichol, R. C., et al. 2006, PhRvD, 74, 063520

Giannantonio, T., Scranton, R., Crittenden, R. G., et al. 2008, PhRvD, 77, 123520

Gorski, K., Hivon, E., Banday, A., et al. 2005, ApJ, 622, 759

Granett, B. R., Kovács, A., \& Hawken, A. J. 2015, MNRAS, 454, 2804

Granett, B. R., Neyrinck, M. C., \& Szapudi, I. 2008, ApJL, 683, L99

Hernández-Monteagudo, C., \& Smith, R. E. 2013, MNRAS, 435, 1094

Ho, S., Hirata, C., Padmanabhan, N., Seljak, U., \& Bahcall, N. 2008, PhRvD, 78, 043519

Hotchkiss, S., Nadathur, S., Gottlöber, S., et al. 2015, MNRAS, 446, 1321

Ilić, S., Langer, M., \& Douspis, M. 2013, A\&A, 556, A51

Klypin, A., Yepes, G., Gottlöber, S., Prada, F., \& Heß, S. 2016, MNRAS, 457, 4340

Kovács, A., \& Granett, B. R. 2015, MNRAS, 452, 1295

Marcos-Caballero, A., Fernández-Cobos, R., Martínez-González, E., \& Vielva, P. 2016, MNRAS, 460, L15

McEwen, J. D., Hobson, M. P., \& Lasenby, A. N. 2008, ITSP, 56, 3813

Nadathur, S. 2016, MNRAS, 461, 358

Nadathur, S., \& Hotchkiss, S. 2015a, MNRAS, 454, 2228

Nadathur, S., \& Hotchkiss, S. 2015b, MNRAS, 454, 889

Nadathur, S., Hotchkiss, S., \& Sarkar, S. 2012, JCAP, 1206, 042

Nadathur, S., Lavinto, M., Hotchkiss, S., \& Räsänen, S. 2014, PhRvD, 90, 103510

Neyrinck, M. C. 2008, MNRAS, 386, 2101

Nolta, M. R., et al. 2004, ApJ, 608, 10

Perlmutter, S., Aldering, G., Goldhaber, G., et al. 1999, ApJ, 517, 565

Planck Collaboration, Adam, R., Ade, P. A. R., et al. 2016a, A\&A, 594, A9

Planck Collaboration, Ade, P. A. R., Aghanim, N., et al. 2014, A\&A, 571, A19

Planck Collaboration, Ade, P. A. R., Aghanim, N., et al. 2016b, A\&A, 594, A21

Reid, B., Ho, S., Padmanabhan, N., et al. 2016, MNRAS, 455, 1553

Riess, A. G., Filippenko, A. V., Challis, P., et al. 1998, AJ, 116, 1009

Sachs, R., \& Wolfe, A. 1967, ApJ, 147, 73

Schäfer, B. M., Pfrommer, C., Hell, R. M., \& Bartelmann, M. 2006, MNRAS, 370, 1713

Szapudi, I., Kovács, A., Granett, B. R., et al. 2015, MNRAS, 450, 288

White, M., Tinker, J. L., \& McBride, C. K. 2014, MNRAS, 437, 2594 